\title{
Full-Mouth Rehabilitation with Metal Ceramic Restoration for Elderly Male Patient: A Case Report with a 30-Month Follow Up
}

Ahmed Ali Ghazwani ${ }^{1}$, Hussam Mousa Muyidi ${ }^{2}$, Mohammed Masoud Mashraqi ${ }^{2}$, Yousef Abdu Gohal ${ }^{2}$, Sameer Jubran Oqayshi $^{2}$, Abubakr Ahmed Siddiq ${ }^{1 *}$

${ }^{1}$ Private Practice, Jazan, KSA

${ }^{2}$ General Practitioner, Ministry of Health, Jazan, KSA

DOI: $10.36348 /$ SJODR.2019.v04i12.005

| Received: 14.11.2019 | Accepted: 20.11.2019| Published: 10.12 .2019

*Corresponding author: Abubakr Ahmed Siddiq

\section{Abstract}

Rehabilitation of a patient with moderate attrition after restoring his vertical dimension is a complex procedure. Therefore, the vertical dimension should be assessed in such a case. This clinical report describes the full-mouth rehabilitation of an elderly patient who was clinically monitored for 30 months to evaluate his adaptation to a removable occlusal splint to restore his vertical dimension. Provisional restorations were then cemented to evaluate the aesthetic and functional outcome to the new occlusion for a time period. Metal ceramic prostheses were cemented. The patient's tolerance to changes in the existing occlusion was confirmed before proceeding to the next steps. During follow-up visits, the patient's family reported a marked improvement in his facial appearance, dental aesthetics, speech articulation, and social life.

Keywords: Metal-ceramic prosthesis, full-mouth rehabilitation, gingivectomy, occlusal splint.

Copyright @ 2019: This is an open-access article distributed under the terms of the Creative Commons Attribution license which permits unrestricted use, distribution, and reproduction in any medium for non-commercial use (NonCommercial, or CC-BY-NC) provided the original author and source are credited.

\section{INTRODUCTION}

Restoration of attrite dentition and missing teeth using cemented metal-ceramic (MC) fixed prostheses is a challenge to dentists and dental technicians. Cemented prostheses offer excellent satisfaction for patients and dentists. Installation of cemented prostheses improves patients' dentition from being unhealthy and unattractive with poor performance into a comfortable and healthy occlusion capable of years of additional services. In addition, it enhances aesthetics, resulting in positive psychosocial changes among patients $[1,2]$.

Moderate dental attrition must be carefully attended to because it not only affects aesthetics but also causes psychological stress to the affected patient. Moreover, it may result in chewing difficulty, temporomandibular joint problems, headaches, pain, and facial collapse. The causes of attrite dentation should be established and managed before any attempt is made to restore it. Attrition may be a result of chemical or mechanical tooth wear or a combination of various factors [3].

For many years, fixed MC prostheses have been the most widely used prosthetics for restoring teeth. Their popularity may be attributed to their clinical durability, oral biocompatibility, and acceptable aesthetics [4]. According to previous studies, the halflife of relatively extensive MC-cemented fixed dental prostheses with proper design is about 15 years $[1,5$, $6]$.

Full-mouth rehabilitation (FMR) involving full arch preparations, impressions, and provisional restorations is regarded as a simultaneous reconstruction. A variety of techniques may be used in simultaneous reconstructions to obtain complete arch dies and mounted casts. These techniques assist in concomitant laboratory construction of the restoration $[1,2,7]$.

Many treatment modalities can be applied for restorations in patients who have lost either maxillary or mandibular teeth, unilaterally or bilaterally, in the presence of attrition in their dentition. Compliance improves when the prosthesis meets the aesthetic requirements of the patient and shows biocompatibility with the oral environment [8]. Patients who take their medicines regularly and properly can be treated as the control. This clinical case report describes a full-mouth dental rehabilitation using $\mathrm{MC}$ prostheses of a controlled medically compromised elderly male patient 
diagnosed with missing teeth and moderate attrite dentition.

\section{CASE REPORT \\ Personal Data}

A 56-year-old male patient reported to the comprehensive care clinic of the College of Dentistry, Jazan University, Kingdom of Saudi Arabia. His main complaints were his small discolored frontal teeth. He consulted to restore his missing and decayed teeth. A multidisciplinary team consisting of a prosthodontist, periodontist, endodontist, and dental ceramist was consulted. He was hypertensive and asthmatic under regular medication. He had several dental treatments in the previous years. The patient did not smoke but did not brush his teeth. Instead, he used miswak to clean his teeth.

\section{Oral and Radiographic Examination}

Extraoral examination revealed a symmetrical face; his lip size and position were normal and competent, and no abnormal findings were observed concerning muscle palpation and soft tissues. Intraoral examination revealed chronic generalized gingivitis and attrition of most teeth. It was severe in maxillary central and lateral teeth. This attrition resulted in slight loss of vertical dimensions and incisal guidance. All teeth were present except numbers 18, 14, 28, 37, and 48. He exhibited Class III molar relationship and group function in both sides. Hard tissue examination revealed caries in teeth numbers $17,12,22,23,44,45,46$, and 47. Tooth number 24 was missing, and fractured tooth number 15 and missing tooth number 36 had been replaced by three united bridges and showed overall generalized attrition (Figs 1A-1E). The panoramic, preapical, and bitewing radiographic interpretation showed mild bone loss, normal anatomy, and position of the condyle of glenoid fossa on both sides. Previous poor RCT in relation to tooth number 15 , restoration of tooth number 27, and three-unit MC bridges was present in the mandibular right side (Figs $1 \mathrm{~F}$ and $1 \mathrm{G}$ ). The general dental chart is presented in Fig-2A. Periodontal propping and pocket depth were recorded (Fig-2B), with both dental plaque and calculus present. The patient diagnosis was chronic generalized gingivitis and generalized attrition; tooth number 23 showed a necrotic pulp with asymptomatic apical periodontitis, and multiple carious teeth were found in both arches.

\section{Treatment Outlines and Options}

The ideal treatment option is presented in Fig3A. The treatment plan developed for the patient is presented in Fig-3B. The whole treatment steps were conducted in four phases according to Rosenstiel et al., [9] and lasted about 3.5 months. The treatment steps were as follows:

Phase I or disease control; Maxillary and mandibular alginate impression and face bow recording of the relation of both arches were conducted. Those casts were mounted on a semi-adjustable Whip-Mix articulator (Waterpik Technologies, Fort Collins, Co, USA) after face bow (Hanau Spring bow) transfer and bite registration through centric and protrusive interocclusal record (Figs 4A-4D). In addition, oral hygiene instruction and motivation, as well as gross scaling and root planning, were performed during different appointments. Excavation of caries and permanent composite restoration were performed for many teeth in both arches using composite resin (Tertic$\mathrm{N}$-Ceramic, Ivoclar Vivaden).

Phase II or surgical phase; Extraction of teeth numbers 17 and 15 was performed. Root canal treatments for teeth numbers $11,21,23,31,32,41$, and 42 using glass fiber posts and composite core were conducted, followed by final coronal restorations. Crown lengthening was performed for maxillary and mandibular anterior teeth (Figs 5A and 5B). An occlusal splint was fabricated and adapted to the patient's maxillary teeth (Fig-5C).

Phase III or rehabilitation phase; All teeth were prepared to receive an MC prosthesis in the form of either separated crowns or a bridge (Figs 6A and $6 \mathrm{~B})$. The maxillary and mandibular final impressions were obtained using an addition silicone (Virtual Ivoclar Vivadent, Lichtenstein) with double mixing techniques (Figs 6C and 6D). At the end of this phase and at this clinical setting, the provisional crowns and bridges were constructed (Success SD, Promedica Neumunster, Germany) and then cemented (Fig-6E) with temporary cementation (Temp-Bond NT, Italy). The final impressions were poured, and dies were made and detached (Figs 7A and 7B). Wax up of the metal coping and metal casting was performed from a nickel chromium casting alloy (Wiron 99, Bego, Germany) following the manufacturer's instruction and conventional laboratory procedure (Figs 7C-7F). The metal copings were fitted in the patient's mouth (Figs 7G-7I). During this clinical step, a shade selection (2M2 - 3D master, shade guide) was carried out for ceramic build up (Figs 7G-7I). At the next appointment, porcelain try-in and occlusal adjustment during centric and eccentric movements were checked and performed, and all prostheses were glazed. The occlusion was adjusted at group function in both sides and then cemented with modified glass ionomer cement (Relaxy, 3M ESPE, Germany; Figs 8A-8D). Postoperative radiographs were obtained (Figs $8 \mathrm{E}$ and $8 \mathrm{~F}$ ).

Phase IV or maintenance phase; The patient was motivated for oral hygiene maintenance, and check-up appointments were made at different time periods. During those visits, he was extremely satisfied with the final restorations. The 30 -month postoperative follow-up appointments demonstrated excellent stability of the restorations, and the patient was free from pain or discomfort with no evidence of jaw discomfort. Positive 
psychosocial changes in the patient's life were conveyed by his wife, sons, and colleagues, as shown in his postoperative intraoral and extraoral views (Figs 9A-9D).

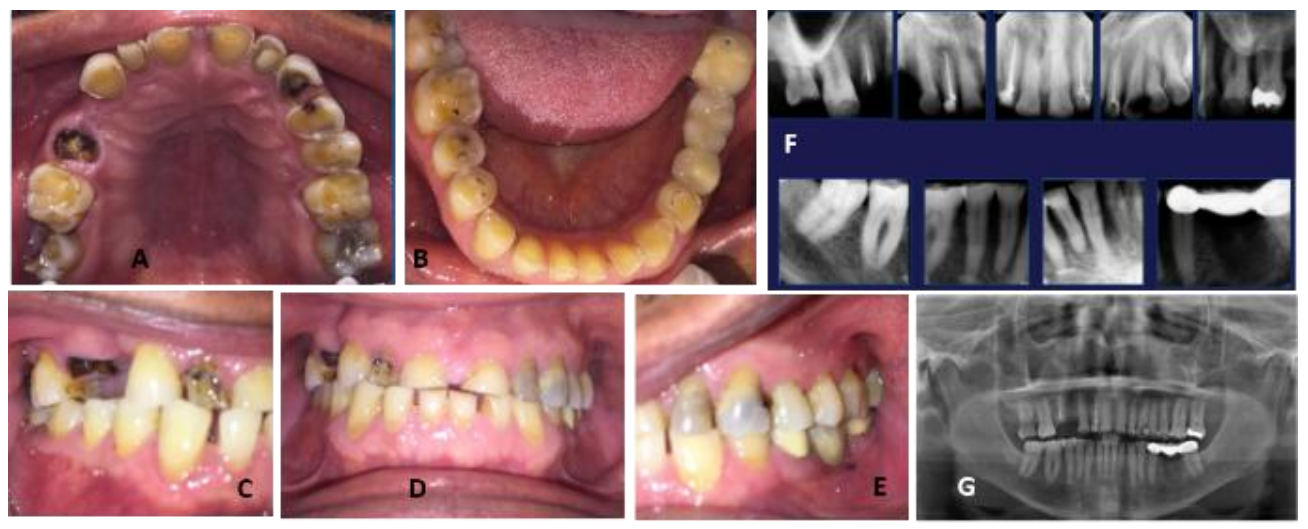

Fig-1: Preoperative intraoral views (A-E); Radiographic views (F and G).

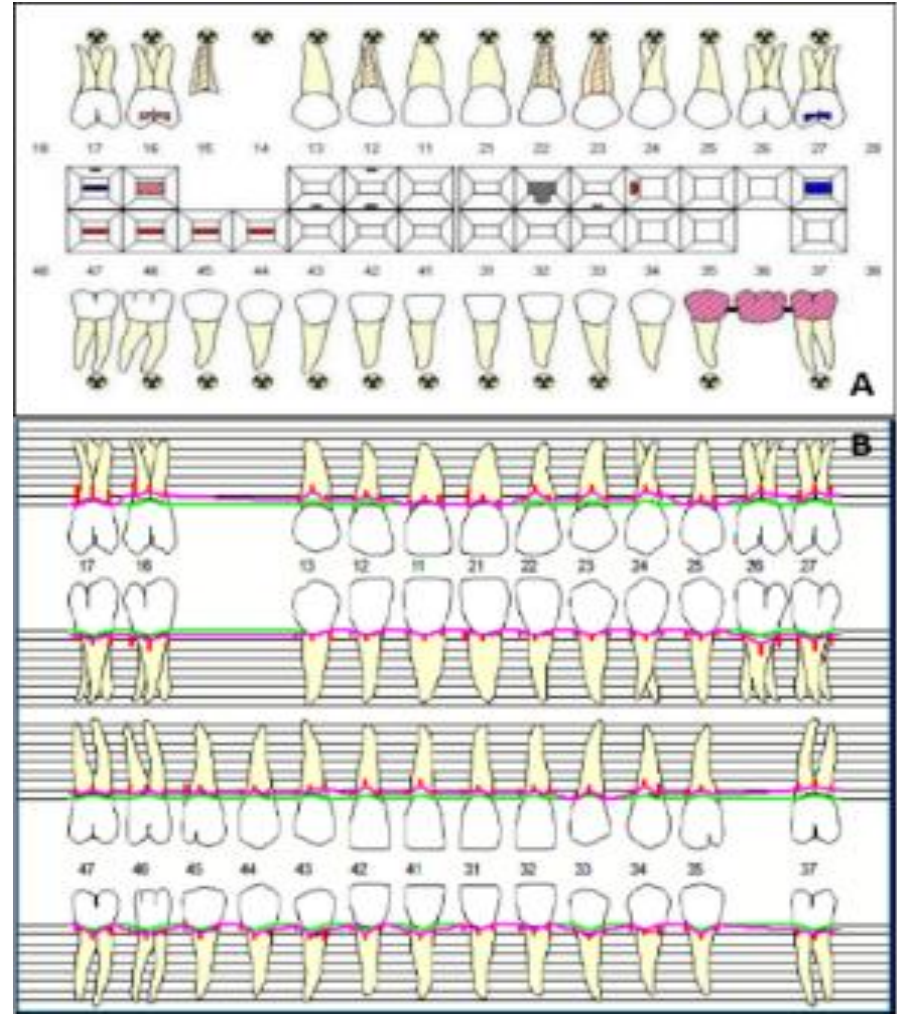

Fig-2: General dental chart (A); Periodontal chart (B)

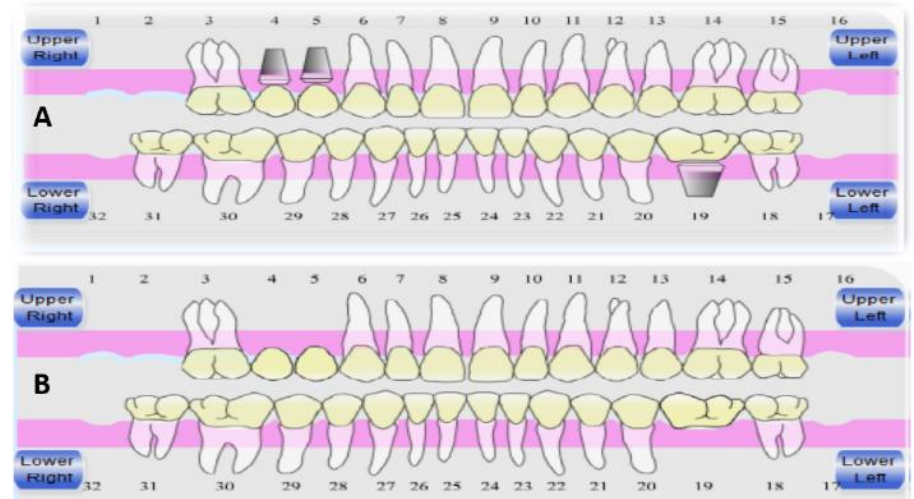

Fig-3: Treatment options (A); Selected treatment (B) 


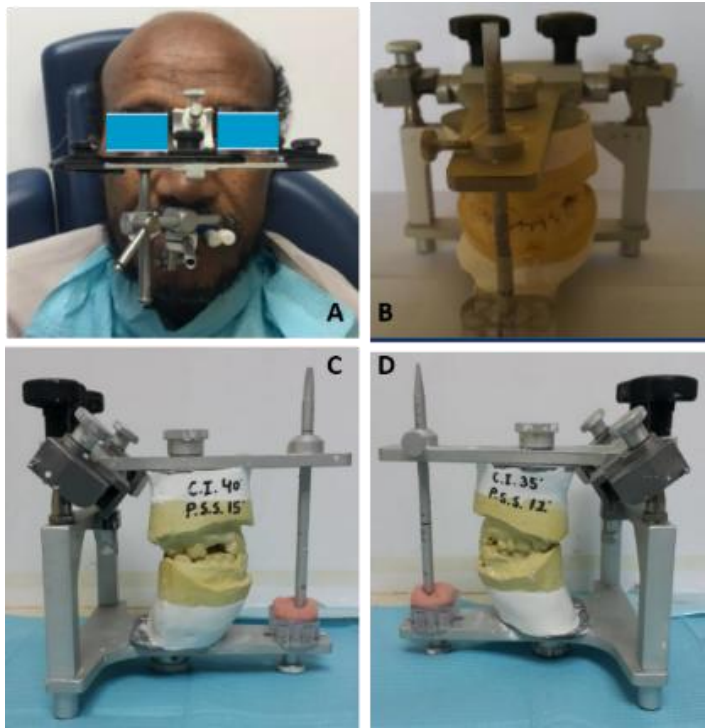

Fig-4: Face bow transfer (A); Mounting casts on articulator with programing (A-D)
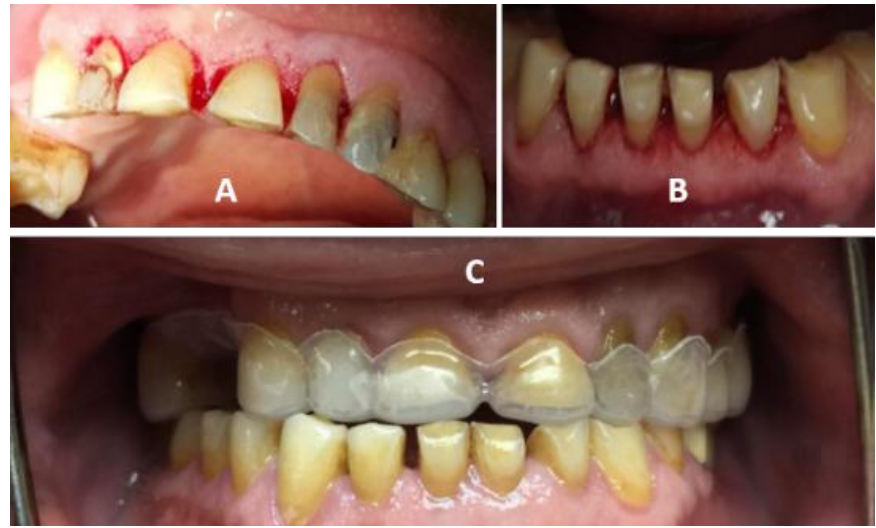

Fig-5: Maxillary and mandibular crown lengthening (A and B); Occlusal splint in place (C)
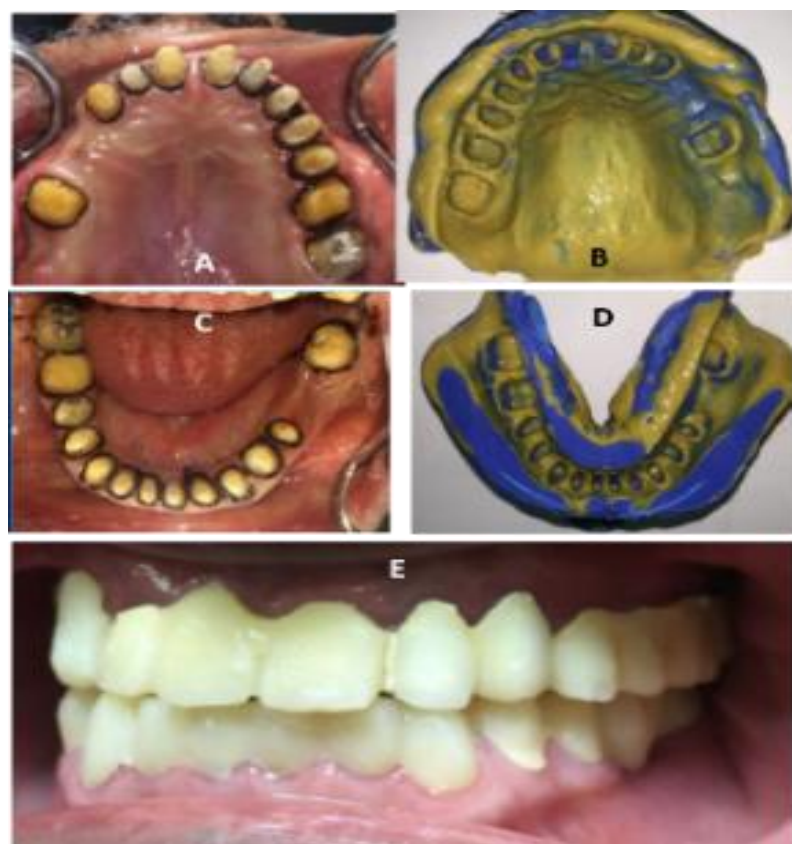

Fig-6: Teeth preparation with retraction cords (A and B); Final impression (C and D); Cemented provisional prostheses (E) 

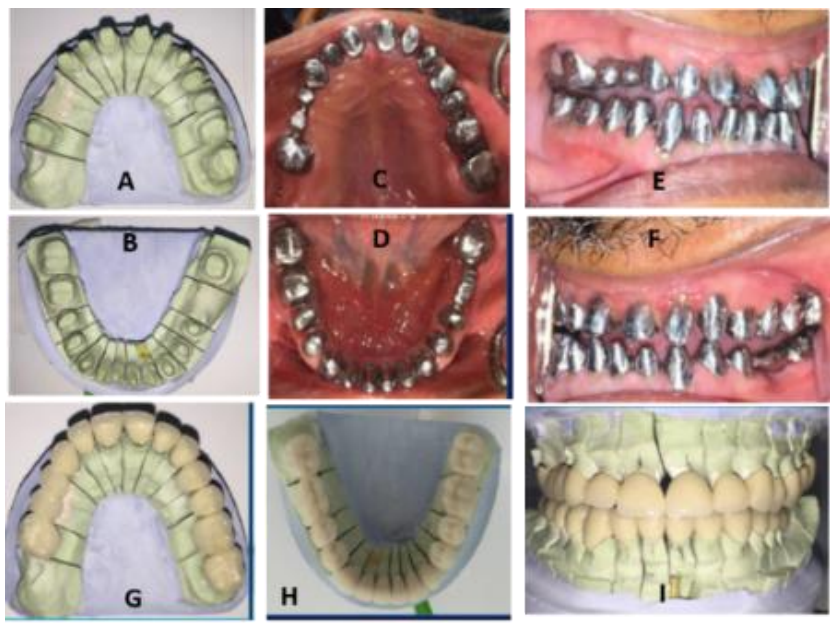

Fig-7: Maxillary and mandibular casts with dies (A and B); During metal try-in (C-F); Finished metal ceramic prostheses (G-I)
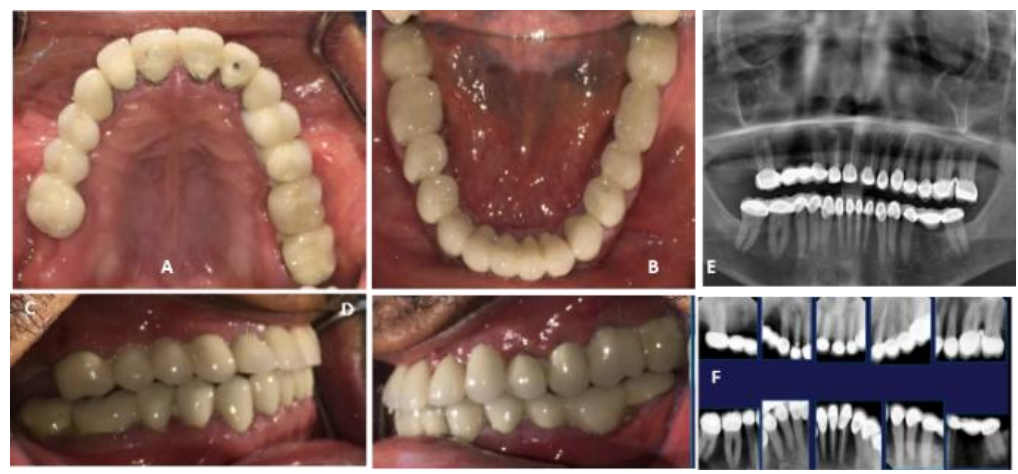

Fig-8: Intraoral views of the cemented metal ceramic prostheses (A-D); Radiographic view of cemented restorations ( $\mathrm{E}$ and $\mathrm{F})$
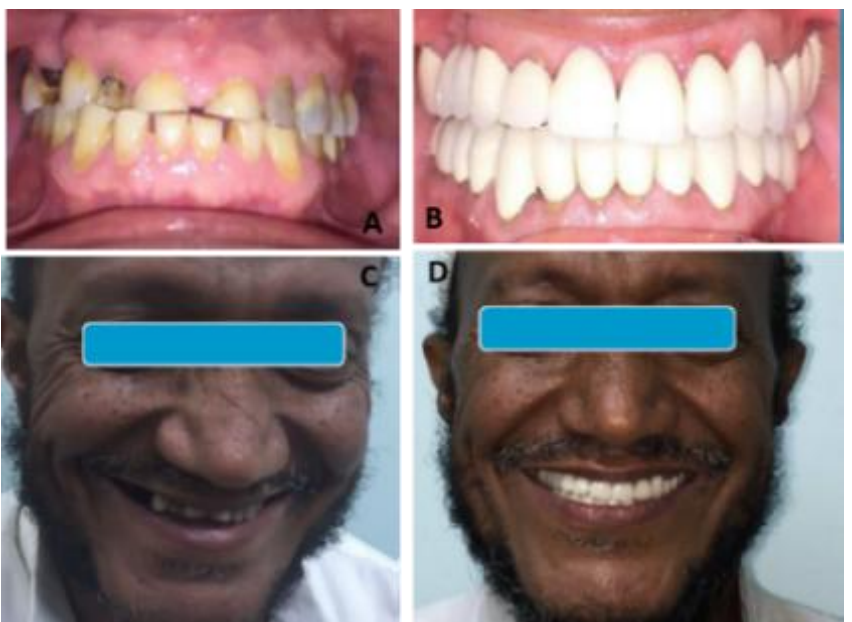

Fig-9: Pre- and post-intraoral views (A and B); Extraoral views (C and D)

\section{DISCUSSION AND CLINICAL SIGNIFICANCE}

FMR restores teeth, bite, and muscles to their original healthy conditions. It helps create a smile that is not only aesthetically pleasing but also functionally comfortable [10]. Rehabilitation of an elderly patient with severely worn dentition after restoring the vertical dimension is a complex procedure. Thus, the vertical dimension should be assessed in such a case. Tolerance to changes in the vertical dimension of occlusion must be confirmed. Articulated study casts and a diagnostic wax up can provide necessary information for evaluating treatment options [1].

The systematic approach for FMR involves several phases. During the diagnostic phase, tolerance toward an increase in occlusal vertical dimensions by using splints is determined. The patient's mental attitude and motivation should also be evaluated. In the 
preparatory phase, an oral hygiene maintenance program and an endodontic treatment plan for tooth diseases and other existing ailments must be developed. In the restorative phase, full-mouth MC crowns are constructed and cemented. In the maintenance phase, all crowns are evaluated at different periods to check prognosis. Moreover, any improvement in the psychological state of the patient should be recorded. We followed these steps in treating our case [9].

Moderate attrition is a result of chemical or mechanical tooth wear or a combination of various factors [11]. The clinical management of aesthetically demanding, complex practical prosthodontic rehabilitation is a challenge. Accurate diagnosis, appropriate treatment planning, prudent selection of materials, and proper treatment execution are essential for a successful treatment outcome over a long period $[6,12]$.

Depending on the degree of tooth attrition, FMR can be accomplished by conservative composite restorations, resin-bonded ceramic crowns, and postcore and cast restorations [13]. In our case, a conservative composite restoration could not be performed because the remaining tooth structures were sufficient for teeth preparation. Moreover, implants could have been used to replace the missing teeth, but our patient had financial constraints and was unwilling to undergo extensive surgical procedures.

MC is still the most widely performed technique for replacing missing tooth/teeth in most clinical conditions. It fulfills the requirements provided by ANSI/ADA Standard No-38 [14]: these requirements are strength of the materials used, familiarity of dental technicians with the technique, biocompatibility, reasonable price, durability, and aesthetic acceptability.

The clinical follow up for this case was more than 2 years. The patient was satisfied with the reconstruction in terms of aesthetics and function. The same outcome was achieved by those followed up their cases for a year or more $[1,8,11,15,16]$.

The management of attrite dentition using FM cemented MC prostheses is complex. Attrite dentition is among the most difficult cases to rehabilitate. Proper assessment of the occlusion dimension is important, and an accurate treatment plan is required in each step for each individual case. Wearing of occlusal splint, followed by temporary cemented FM provisional prosthesis can provide important information to ensure the success of the selected treatment option. In our case, tolerance to minor changes in VDO was confirmed with clinical evaluation of the patient after having worn a diagnostic splint and provisional prosthesis.

\section{CONCLUSION}

An ideal aesthetic treatment plan attempts to achieve perfection in every way. A range of treatment options must be reviewed to achieve an aesthetic smile and proper function while maintaining a healthy oral environment. Recognizing that form follows function and that the anterior teeth play a vital role in maintaining oral health are paramount. In this clinical report, raising VDO using a removable occlusal splint followed by MC restorations was based on accurate diagnosis to ensure the successful FMR for moderate attrite dentition.

\section{REFRENCES}

1. Jain, A. R., \& Ariga, P. (2013). Full mouth rehabilitation of a patient having limited interarch space with manbibular implant retained fixed adoro fused to metal Fp-1 prosthesis and maxillary acrylic removable conventional complete denture. Int J Oral Implantol Clin Res, 4(3), 112117.

2. Jain, A. R., Nallaswamy, D., \& Padma Ariga, J. M. P. (2013). Full mouth rehabilitation of a patient with reduced vertical dimension using multiple metal ce ramic restorations. Contemporary clinical dentistry, 4(4), 531-535.

3. Bansal, R., Jain, A., Mittal, S., \& Kumar, T. (2014). Full mouth rehabilitation in a medically compromised patient with fluorosis. Journal of clinical and diagnostic research: JCDR, 8(7), ZD22-ZD24.

4. Trautmann, G., Gutmann, J. L., Nunn, M. E., Witherspoon, D. E., \& Shulman, J. D. (2000). Restoring teeth that are endodontically treated through existing crowns. Part II: Survey of restorative materials commonly used. Quintessence International, 31(10):719-728.

5. Jain, A. R., Nallaswamy, D., \& Padma Ariga, J. M. P. (2013). Full mouth rehabilitation of a patient with mandibular implant screw retained Fp-3 prosthesis opposing maxillary acrylic removable over-denture. Contemporary clinical dentistry, 4(2), 231-235.

6. Jain, A. R. (2014). Fp1 Prosthesis in Maxillary Ridge Defect and Fixed Partial Denture in Mandibular Ridge Defect-A Case Report. International Journal, 2(6), 184-189.

7. Hobo, S., \& Takayama, H. (1997). Twin stage procedure, Part 2: A clinical evaluation test. Int Periodont Rest Dent, 17: 457-63.

8. Jain, A. R. (2018). Full mouth rehabilitation of a patient using multiple metal ceramic restorations: A case report. Drug Invention Today, 10(5): 6736.

9. Rosenstiel, S. F., Land, M. F., \& Fujimoto, J. (2015). Contemporary Fixed Prosthodontics, 5th ed. St. Louis Mosby Elsevier; Planning and Preparation; 70, 138-158. 
10. Dawson, P. E. (2007). Functional Occlusion: From TMJ to Smile Design. 1st ed. Mosby St. Louis, Elsevier.18-26.

11. López-Frías, F. J., Castellanos-Cosano, L., MartínGonzález, J., Llamas-Carreras, J. M., \& SeguraEgea, J. J. (2012). Clinical measurement of tooth wear: Tooth wear indices. Journal of clinical and experimental dentistry, 4(1), e48-e53.

12. Jain, A. R., \& Janani, T. (2016). Full mouth rehabilitation of an ectodermal dysplasia patient with hypodontia and reduced vertical dimension using metal ceramic restorations: a case report. Biology and Medicine, 8(6): 335-340.

13. Smales, R. J., \& Berekally, T. L. (2007). Longterm survival of direct and indirect restorations placed for the treatment of advanced tooth wear. European Journal of Prosthodontics and Restorative Dentistry, 15(1):2-8.

14. ANSI/ADA Standard No (38). Dental Products: Standards, Technical Specifications and 1 Reports. Metal-Ceramic Dental Restorative Systems: 2000 (Reaffirmed 2015) ISO 9693:1999.

15. Qahhar, M. A. W., Alkhayrat, F. M., \& Hakami, B. M. (2016). A One Year Follow-Up of a Full Mouth Rehablitation for Severly Attrited Dentition. Saudi Journal Oral Dental Research, 1(2):58-63.

16. Hegde, C., Krishna, D. P., Jacob, S. J., \& Shetty, M. (2009). Full mouth rehabilitation of a severely worn out dentition to functional harmony. The Journal of Indian Prosthodontic Society, 9(3), 164-166. 